\title{
Reliability and Uniformity Enhancement in 8T-SRAM based PUFs operating at NTC*
}

\author{
Pramesh Pandey, Asmita Pal, Koushik Chakraborty, Sanghamitra Roy \\ USU BRIDGE LAB, Electrical and Computer Engineering Department, Utah State University \\ Logan, Utah, USA \\ \{pandey.pramesh1,asmita.pal\}@aggiemail.usu.edu,\{koushik.chakraborty,sanghamitra.roy\}@usu.edu
}

\begin{abstract}
SRAM-based PUFs (SPUFs) have emerged as promising security primitives for low-power devices. However, operating 8T-SPUFs at Near-Threshold Computing (NTC) realm is plagued by exacerbated process variation (PV) sensitivity which thwarts their reliable operation. In this paper, we demonstrate the massive degradation in the reliability and uniformity characteristics of 8T-SPUF. By exploiting the opportunities bestowed by schematic asymmetry of 8T-SPUF cells, we propose biasing and sizing based design strategies. Our techniques achieve an immense improvement of more than $55 \%$ in the percentage of unreliable cells and improves the proximity to ideal uniformity by $82 \%$, over a baseline NTC 8T-SPUF with no enhancement.
\end{abstract}

\section{CCS CONCEPTS}

- Security and privacy $\rightarrow$ Security in hardware; Embedded systems security; $\bullet$ Hardware $\rightarrow$ Integrated circuits;

\section{KEYWORDS}

6T-SRAM,8T-SRAM, PUF, Near Threshold, Hardware security

\section{ACM Reference Format:}

Pramesh Pandey, Asmita Pal, Koushik Chakraborty, Sanghamitra Roy. 2018. Reliability and Uniformity Enhancement in 8T-SRAM based PUFs operating at NTC. In ISLPED '18: ISLPED '18: International Symposium on Low Power Electronics and Design, July 23-25, 2018, Seattle, WA, USA. ACM, New York, NY, USA, 6 pages. https://doi.org/10. $1145 / 3218603.3218642$

\section{INTRODUCTION}

SRAM-based PUFs (SPUFs) have emerged as a viable security choice in resource constrained systems [3]. This can be

\footnotetext{
*This work was supported in part by National Science Foundation grants (CAREER-1253024, CCF-1318826, CNS-1421022, and CNS-1421068). Any opinions, findings, and conclusions or recommendations expressed in this material are those of the authors and do not necessarily reflect the views of the National Science Foundation.
}

Permission to make digital or hard copies of all or part of this work for personal or classroom use is granted without fee provided that copies are not made or distributed for profit or commercial advantage and that copies bear this notice and the full citation on the first page. Copyrights for components of this work owned by others than ACM must be honored. Abstracting with credit is permitted. To copy otherwise, or republish, to post on servers or to redistribute to lists, requires prior specific permission and /or a fee. Request permissions from permissions@acm.org.

ISLPED '18, July 23-25, 2018, Seattle, WA, USA

(C) 2018 Association for Computing Machinery.

ACM ISBN 978-1-4503-5704-3/18/07 .\$15.00

https://doi.org/10.1145/3218603.3218642 attributed to their obviation for dedicated circuitry and elimination of the overheads of complex encryption mechanisms [17]. SPUFs rely on inherent physical characteristics, that originate from manufacturing process variations (PV), to enable chip security $[8,9,17]$. Likewise, Near-Threshold Computing (NTC) has transpired as a promising energy-efficient design paradigm, as compared to Super Threshold Computing (STC) [6]. However, the supply voltage reduction is also accompanied by increasing effects of PV. Herein, it is important to highlight a unique property of SPUFs to reproduce the same chip signature every time it is attempted to be authenticated, often referred to as SPUF reliability [9]. Thus, it remains an intriguing research question whether NTC operation of SPUFs brings about any degradation in reliability, due to exacerbated PV sensitivity.

The read instability introduced by low voltage operation makes 6T-SRAMs an unfavorable design choice at NTC [6], and the addition of extra read transistors in 8T-SRAM used in NTC [4], introduces a schematic asymmetry, quite contrary to a symmetrical 6T-SRAM. We observe that, this leads to an asymmetric start-up current, which when sensitized by varying system noise and temperature, leads to a degradation in SPUF reliability. In addition to reliability concerns, we find that the shift to NTC SPUFs challenges ideal SPUF uniformity owing to the differences in device geometry. SPUF uniformity depicts how uniformly the 0 's and 1's are distributed in the SPUF signature [7]. We observe that the schematic asymmetry exhibited by 8T-SPUFs leads to an imbalanced distribution of the start-up current within the cell, giving rise to decreased uniformity. We analyze the impact of device asymmetry on reliability and uniformity of 8T-SPUF and propose CUBIT: Biasing based strategies and CUSIT: Sizing based strategies.

Our contributions in this paper are as follows:

- We observe that there is a marked degradation in reliability and uniformity for 8T-SPUFs operating at NTC, in comparison with STC-operated 6T-SPUFs (Section 2).

- By analyzing the impact of device asymmetry on reliability and uniformity characteristics, we propose CUBIT: biasing based design strategy, and CUSIT: sizing based design strategy (Section 3).

- In comparison to state-of-the-art technique by Chang et al. [3], our proposed design strategies exhibit a comprehensive enhancement in both reliability and uniformity, with more than $55 \%$ improvement in percentage of unreliable cells, and $82 \%$ progression in the ballpark of ideal uniformity over the Baseline NTC 8T-SPUF array (Section 4). 


\section{BACKGROUND AND MOTIVATION}

In this section, we quantify the metrics of reliability (Section 2.1) and uniformity (Section 2.2), which are key determinants of SPUF behavior. Using the methodology discussed in Section 2.3, we demonstrate that although 8T-SPUFs exhibit an energy efficient solution [6], their naive implementation on low power platforms come with massive degradation in reliability and uniformity (Section 2.4).

\subsection{Estimating SPUF Reliability}

SPUF Reliability is a measure of repeatability of SPUF array signature. The reliability is threatened when the start-up states of cells in the unique signature of SPUF array are flipped by noise and environmental variations, leading to unreliable cells [9]. In Equation (1), Bit_Flips gives the number of times an SPUF cell $c$ powers up to a different bit value, relative to the noiseless iteration. $n$ is the number of power-ups of the same SPUF cell in the presence of system noise.

$$
\text { Bit_Flips }\left(B F_{C}\right)=\sum_{i=0}^{n} \mid \text { Bit_value }_{\text {noiseless }}-\text { Bit_value }_{i} \mid
$$

Bit_Reliable is a binary thresholder, determining whether the $c$-th SPUF cell is reliable. $t$ is the threshold of number of allowed bit flips to still mark the cell as reliable.

$$
\text { Bit_Reliable }\left(B R_{c}\right)= \begin{cases}1 & B F_{c} \leq t \\ 0 & B F_{c}>t\end{cases}
$$

Finally, PUC, gives the Percentage of Unreliable Cells in the SPUF with $m$ cells, at supply voltage $V$ and temperature $T$.

$$
\operatorname{PUC}_{(V, T)}=\frac{1}{m} \sum_{c=0}^{m}\left(1-B R_{c}\right) \times 100 \%
$$

For assessing reliability in this paper, we set $t=0, n=10, m=1000$.

\subsection{Estimating SPUF Uniformity}

Uniformity depicts the randomness of the SPUF array's signature. Uniform distribution of ' 1 's and '0's in the SPUF signature ensures a strongly random key, which is difficult to be replicated by an attacker [7]. For a m-bit SPUF, Maiti et al. have defined uniformity as the percentage Hamming Weight (HW) of the m-bits [13], given by Equation (4).

$$
\text { Uniformity }(\%)=\frac{1}{m} \sum_{c=0}^{m} \text { Bit_value }_{c} \times 100 \%
$$

where Bit_value is the power up state of the $c$ th SPUF cell ('0' or ' 1 ') of SPUF with $m$ cells. A uniformity proximal to $50 \%$ translates to more randomness in the SPUF.

\subsection{Methodology}

To estimate the reliability and uniformity of an SPUF array, we model 6T-SRAM and 8T-SRAM cells using Predictive Technology Model (PTM) for 32nm [1] We instantiate 1000 unique cells to build an SPUF array by Monte Carlo simulations for Threshold voltage $\left(V_{t h}\right)$, Length (L) and Width (W), with PV of $9 \%$ for $V_{t h}$ and $4.5 \%$ for $\mathrm{W}$ and L. We model the noise in time-domain using all the noise sources defined in BSIM-CMG
[12]. For the frequency-domain noise modeling, the base and maximum frequency are set to $F_{\text {min }}=10^{4}$ and $F_{\text {max }}=10^{9}$ respectively [18]. We then simulate the SPUF array at supply voltage ranging from $-10 \% V_{d d}$ to $+10 \% V_{d d}$ and temperature from $-40^{\circ} \mathrm{C}$ to $110^{\circ} \mathrm{C}$.

\subsection{Results and Significance}

Figure 1a shows the comparison of PUC in STC 6T-SPUF and NTC 8T-SPUF array. We observe that the PUC variation across $V_{d d}$ is under $5 \%$ for both the STC and NTC SPUF. However, the window of variation of PUC across temperatures for NTC 8T-SPUF shoots up to $30 \%$, as opposed to $6 \%$ in STC 6T-SPUF. Fuzzy extractor and other error correcting mechanisms would have to cover a larger spread of unreliable cells across all corners of environmental variations, which leads to excessive power and area overheads [16]. The increased overheads will disrupt the entire SPUF ecosystem, which is primarily targeted for low cost security primitives. Hence, shifting from STC to NTC, SPUFs subjected to temperature variation, are plagued by decreased reliability.

Figure $1 \mathrm{~b}$ compares the uniformity of STC 6T-SPUF and NTC 8T-SPUF at different temperatures. We see that the worst case deviation of 6T-SPUF from ideal uniformity is under $9 \%$, as compared to the glaring deviation of $33.2 \%$ for $8 \mathrm{~T}-\mathrm{SPUF}$. This anomaly can be attributed to the exorbitant skewness of the bits to a particular state for the NTC 8T-SPUF. Therefore, it is imperative to realise that the 8T-SPUFs are plagued by decrement in uniformity, making them more vulnerable to attacks. To recuperate this atrophy in reliability and uniformity characteristics, we propose our design strategies, for SPUFs operated at NTC in Section 3.

\section{DESIGN}

In this section, we first discuss the impact of schematic asymmetry in 8T-SPUFs amalgamated with the increased effect of $\mathrm{PV}$, to justify the governing principle of our design (Section 3.1). Following which, we propose our design strategies, $\mathrm{CU}-$ BIT: Current Suppression with Biasing Technique (Section 3.2) and CUSIT: Current Suppression with Sizing Technique (Section 3.3), to tackle the glaring degradation in reliability and uniformity characteristics of 8T-SPUFs at NTC.

\subsection{Impact of Schematic Differences}

The schematic difference between a 6T-SPUF and 8T-SPUF cell is the addition of the two NMOS's for read access. Schematically, as the read access transistors are only connected to one half of the cell (Fig. 2), there is an asymmetry in right half and left half supply current, $I_{R H}$ and $I_{L H}$ (Fig.3).

We compare the maximum of currents, $I_{R H}, I_{L H}$ among 10 different noisy startups, until the trip point [9], from where the voltages at $B L$ and $B L B$ diverge to their final states. Figure 4a shows that the current $I_{R H}$ dominates the current $I_{L H}$. This non-uniformity in current distribution among the SPUF cells is brought about by gate leakage current, $I_{g}$ flowing from right junction $J R$ towards the gate of transistor M7 as shown in Figure $3 . I_{g}$ tries to drag down the voltage rise at junction $J R$. 


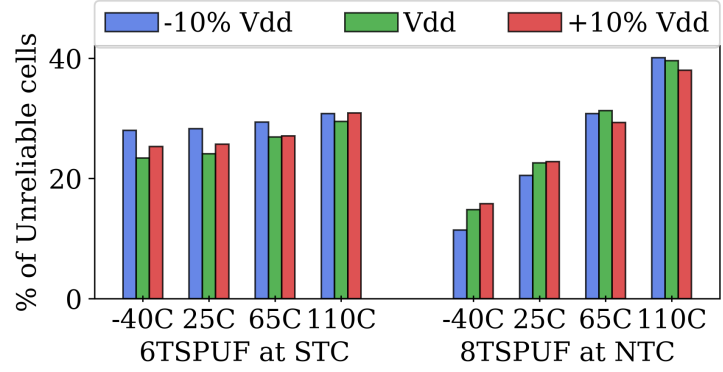

(a) PUC comparison w.r.t. temperature and $V_{d d}$ fluctuations

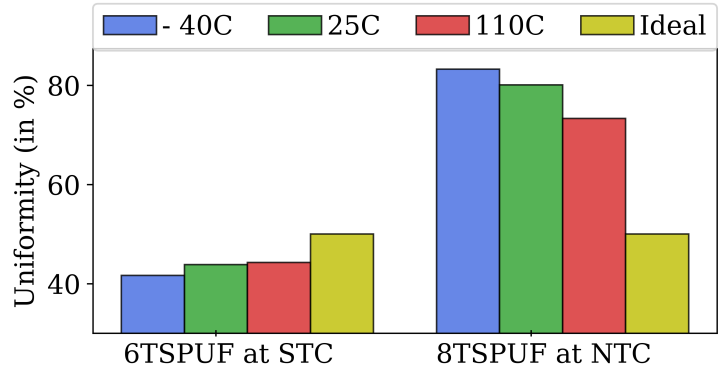

(b) Uniformity $(\%)$ comparison w.r.t. temperature variations

Figure 1: Reliability and Uniformity characteristics for STC-operated 6T-SPUF versus NTC-operated 8T-SPUF

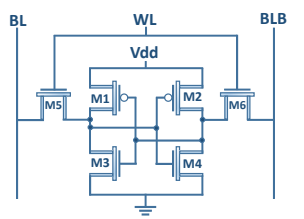

(a) 6T-SPUF cell
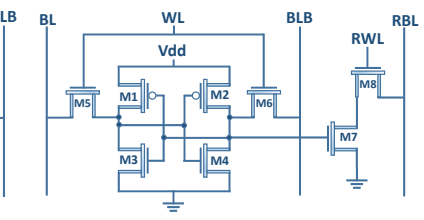

(b) 8T-SPUF cell
Figure 2: Schematic Representation of a SPUF cell

This phenomenon leads to more number of SPUF cells ending up to a final states ' 1 ' than ' 0 ' at $J L$, .i.e degraded uniformity. In addition, we observe that this current is very sensitive to temperature change and random system noise. Due to this increased sensitivity, the chances of degraded reliability increases manifold. Hence, the suppression of this current and its variation opens doors to better reliability and uniformity in NTC 8T-SPUF. In CUBIT, we demonstrate how biasing can improve the reliability by suppression of $I_{g}$. Following which, we present CUSIT, which is based on sizing to improve the uniformity by $I_{g}$ suppression.

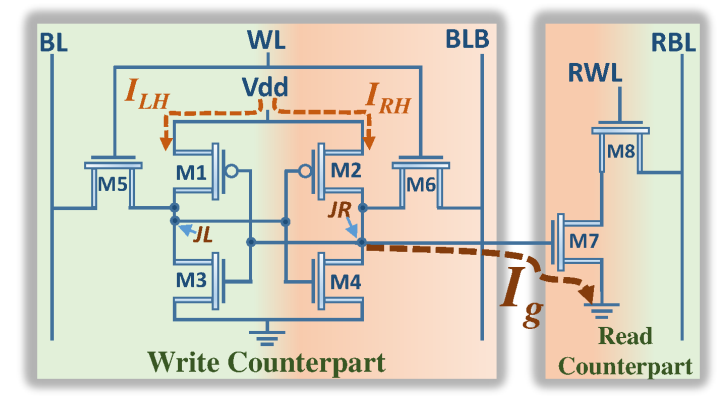

Figure 3: Current Ig is shared from only right junction JR of the $8 T-S P U F$ cell, rendering the current in the right half, $I_{R H}$ asymmetric to left half current $I_{L H}$.

\subsection{CUBIT: Biasing based Techniques}

CUBIT improves the reliability and uniformity of the NTC 8T-SPUF by biasing Read Counterpart of NTC 8T-SPUF (Fig. 3 ) in different ways, targeting the suppression of current Ig. In Figure $4 b$, we show the supression of maximum of current
Ig in SPUF cells by one of our biasing technique, which improves both reliability and uniformity. Similarly, in Figure 4c, we show the massive reduction of statistical variance of maximum of current $I g$ among the noisy startups for SPUF cells for the same technique. Higher magnitude and variance contributes towards decreased reliability and non uniformity respectively. Different biasing techniques are discussed in 3.2.1, as different steps of an algorithm, devised to comprehensively improve both reliability and uniformity.

3.2.1 CUBIT Algorithm. We propose an algorithm for finding the best combination among the different ways of biasing Read Counterpart (Fig. 3). At the end of the algorithm, we are presented with a stack of improved uniformity and reliability figures and the respective moves which cause it. Top of the stack is the best combination according to priority constraints given. User can also select sub optimal solutions as a tradeoff with the overheads in actual implementation. We start by applying minimum possible moves first, and then move to different combinations of moves. Table 1 lists the terminologies and the objective of our algorithm 1.

\begin{tabular}{|l|l|}
\hline T & $\left\{-40^{\circ} \mathrm{C}, 25^{\circ} \mathrm{C}, 110^{\circ} \mathrm{C}\right\}$ \\
\hline R & Max $(\%$ of Unreliable Cells (PUC)), across T \\
\hline U & Max(Uniformity-Ideal Uniformity), across T \\
\hline RLT & Reliability Loss Threshold: Maximum allowed Loss of R (in \%) to gain U. \\
\hline ULT & Uniformity Loss Threshold: Maximum allowed Loss of U (in \%) to gain R. \\
\hline Objective & Minimize U and R \\
\hline
\end{tabular}

Table 1: Terminologies and Objective for Algorithm 1

Different moves for the algorithm are: 1. RE-RBL: This is a Reliability Enhancer move, where we bias the Read Bit Line (RBL) of the 8T-SPUF cell to Biasing voltage (VB), as shown in Fig. 6a. The SPUF array logic can be customized to provide a logic high through precharging in RBL line at the startup of the 8T-SPUF array. Simulation results in Fig. 5, show that, with $\mathrm{VB}=\mathrm{Vdd}$, this move can decrease $\boldsymbol{R}$ (Fig. 5a), by $55 \%$, but cannot decrease $U$ (Fig. 5b).

2. RE-RWL: This is also a RE move, where we bias the Read Word Line (RWL) of the 8T-SPUF cell to VB as shown in Fig. 6b. The SPUF array logic can be customized to enable RWL, also at the startup. Simulation results in Fig. 5, show that, with $\mathrm{VSB}=\mathrm{Vdd}$, this move can decrease $\boldsymbol{R}$ (Fig. 5a), by $53 \%$, but cannot decrease $U$ (Fig. 5b). 


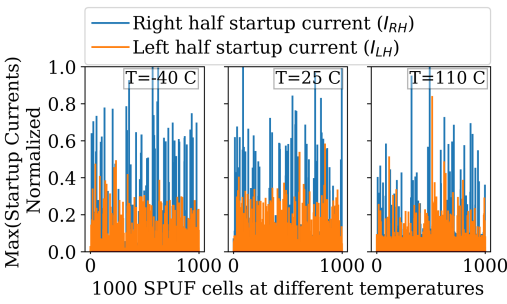

(a) Asymmetric Distribution of startup current

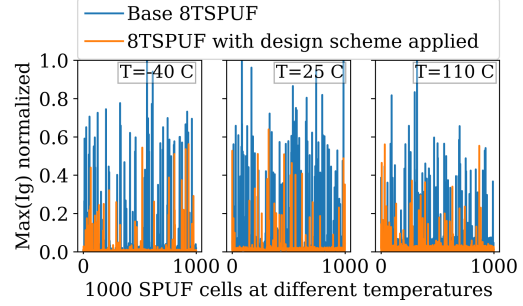

(b) Suppression of Ig Magnitude

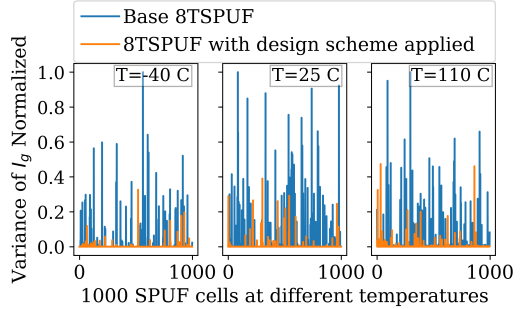

(c) Suppression of Ig Variance

Figure 4: Fig. 4a: Plot of the maximum supply currents distributed to right and left half of 8T-SPUF cell. Fig. $4 b$ : Effective suppression of Ig by biasing techniques. Fig. 4c: Effective supression of Variance of Ig by biasing techniques. Maximum and variance are calculated among the maximum currents until trip point, of 10 different noisy startups

3. RUE-SGT and UE-SGTf: Biasing the Source Ground Terminal (SGT) of M7 with voltage $\mathrm{VB}=\mathrm{Vdd}$, as shown in Fig. $6 c$, at the startup of the 8T-SPUF cell, gives us Reilability and Uniformity Enhancher (RUE) move. As the sink of the current $I g$ is SGT, rising its potential from ground supresses the $I g$ very effectively. Simulation results in Fig. 5 show that bias of $\mathrm{VSB}=\mathrm{Vdd}$ at SGT is able to reduce $R$ and $U$ by $55.8 \%$ and $56 \%$ respectively. RUE-SGT improves the uniformity by aggressively turning around the population of ' 1 ' skewed cells to from $83 \%$ (67\% above ideal) to 35\% (30\% below ideal). Hence, by reducing the degree of supression with lowering VB from Vdd, the uniformity can be brought closer to ideal uniformity. This give us Uniformity Enhancher (UE) moves, UE-SGTf, where $f$ is $\mathrm{VB}$ as fraction of cell $\mathrm{Vdd}(V B / V d d)$. Simulation results in Fig. 5b shows improvement of $\boldsymbol{U}$ with decrease of VB. However, as the VB goes on decreasing, reliability decreases severely as shown in Fig. 5a. Hence, true benefit of UE-SGTfs can be extracted only by coupling with REs.

\subsection{CUSIT: Sizing based Techniques}

CUSIT is a sizing based technique to supress the effects of current $I_{g}$, for better reliability and uniformity. CUSIT scales the sizes of the transistors of $I_{g}$ source (Write counterpart) and sink (Read counterpart) in such a way that $I_{g}$, relative to supply current, $I\left(V_{d d}\right)$ is decreased. This scaling ensures that the impact of $I_{g}$ on reliability and uniformity is curtailed. Figure 7 shows the variation in $I_{g}$ for upscaling write transistors relative to read transistors, with six different scaling factors. $I_{g}$ is normalized to $I\left(V_{d} d\right)$ and observed till the time a 8T-SPUF reaches its trip point. It is evident from the figure that $I_{g}$ decreases with an increase in the upscaling factor. To establish the adaptive nature of CUSIT in the light of varying currents in the transistor, we present two different approaches to transistor resizing. First, scaling down the size of read transistors (relative to write transistors), and second, scaling up the size of write transistors (relative to read transistors). In light of implementation feasibility, the Read and Write Counterparts of 8T-SPUF cells (Fig. 3) can be sized independent of each other, unlike the meticulous sizing constraints of conventional 6T-SPUF cells [2].

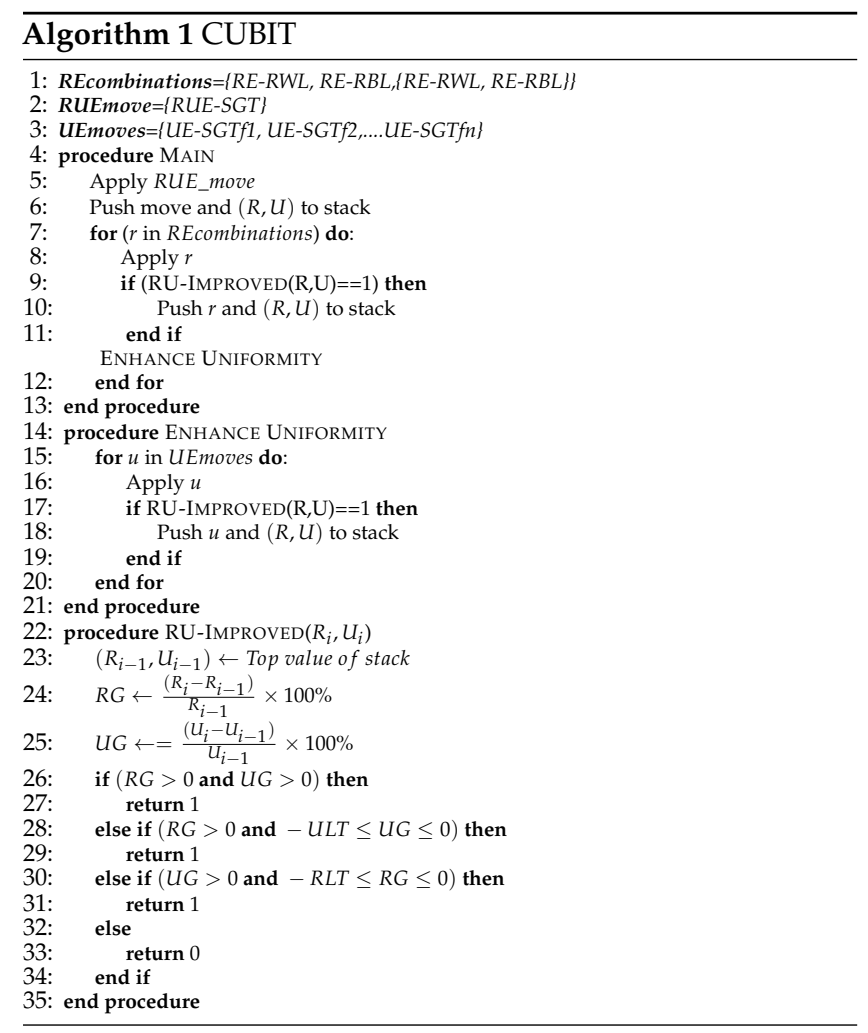

\section{RESULTS}

Section 4.1 presents the results obtained by applying CUBIT and Section 4.2 presents results obtained by aplying CUSIT, comparing it with a comparative sizing scheme in literature.

\subsection{CUBIT Results}

In this section, we present and analyze the results of applying CUBIT algorithm. Table 2 shows the final combination of RE/RUE/UE moves which minimize the $(R, U)$ with different priority constraints. We are able to obtain an excellent enhancement of more than $(51 \%, 76 \%)$ in $(R, U)$ across the Baseline NTC 8T-SPUF across all priority constraints. We observe that the optimal combination of biasing techniques for best Reliability and Uniformity is a trade-off. If we favor Reliability (Uniformity), we get 55.8\% (82.86\%) enhancement in 


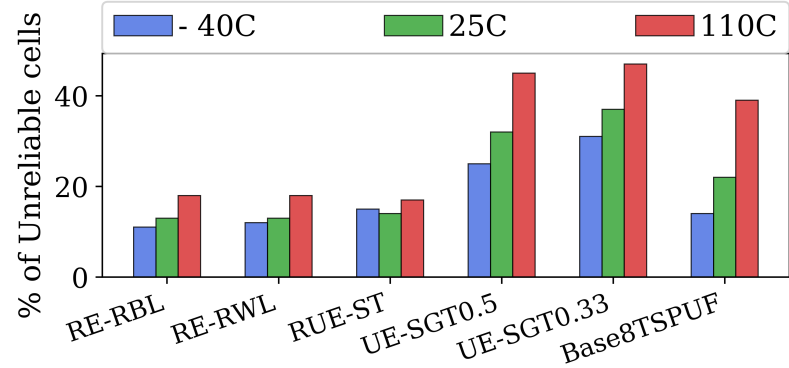

CUBIT Algorithm Biasing Moves

(a) Comparison of Reliability Improvement

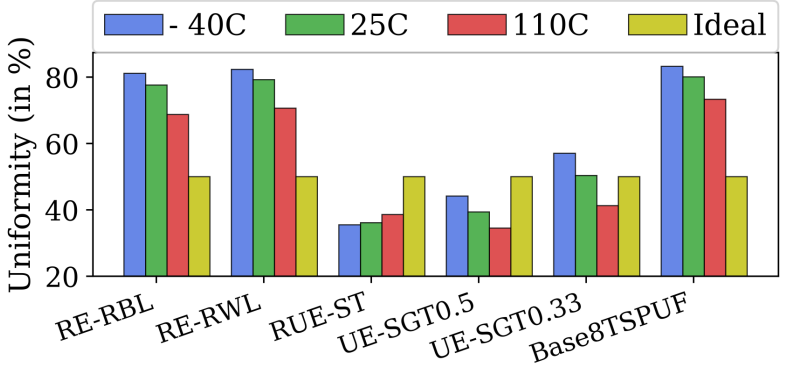

CUBIT Algorithm Biasing Moves

(b) Comparison of Uniformity Improvement

Figure 5: Improvement in Reliability (Fig. 5a) and Uniformity (Fig. 5b) obtained by different biasing schemes. Individual biasing schemes cannot always address comprehensive improvement in both reliability and uniformity.

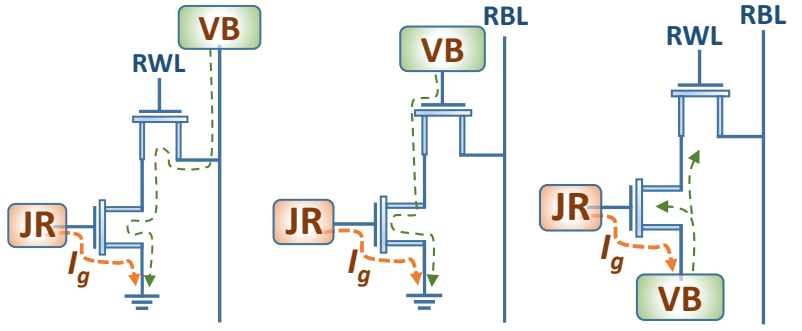

(a) Biasing RBL with VB (b) Biasing RWL with VB (c) Elevating GND to VB

Figure 6: Moves to effectively supress the magnitude and/or variation of the current Ig, which bias a voltage VB at different terminals of Read Section of 8T-SPUF cell in CUBIT Algorithm.

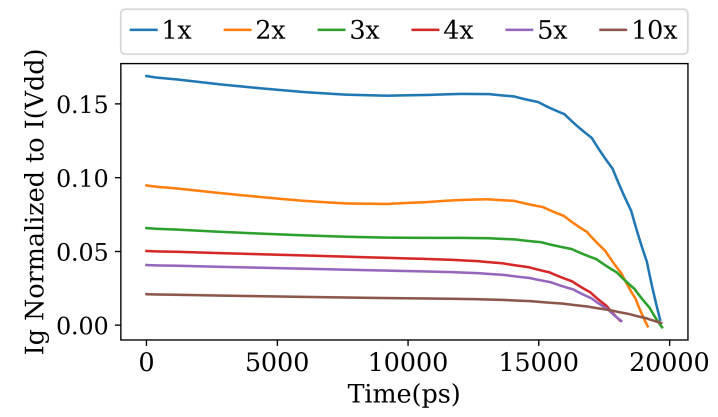

Figure 7: Supression of normalized current Ig with different size upscaling factors of CUSIT

$\mathrm{R}(\mathrm{U})$. Also, even by only using a single voltage source which eliminates the need of additional low voltage source, we get $(54.9 \%, 76.8 \%)$ enhancement in $(\mathrm{R}, \mathrm{U})$ as compared to Baseline 8T-SPUF.

\subsection{CUSIT Results}

In this section, we present the improvement in reliability obtained with CUSIT. As we do not have a sizing technique for 8T-SPUF's quality improvement in literature, we compare CUSIT with a sizing technique [3] demonstrated for 6T-SPUFs which holistically upscales the size of all transistors of SPUF cell targeting Vth variation (VCTS-Vth Centric Transistor Sizing). For CUSIT, we upscale the size of transistors in write counterpart relative to the size of transistors in read counterpart, targeting supression of current Ig. Table 3 shows that reliability improvement with size upscaling is similar in both techniques, achieving improvement in PUC by more than $50 \%$ when upscaling factor reaches 5 . However, CUSIT vastly outperforms VCTS in terms of comprehensive enhancement of both reliability and uniformity as CUSIT achieves more than $72 \%$ enhancement in proximity to ideal uniformity, compared to an insignificant $3 \%$ improvement by VCTS. VCTS cannot achieve uniformity improvement because, holistic scaling of all the transistors with a common factor can't reduce the effect of asymmetric current $I g$. Hence, it is imperative to deduce that CUSIT is a superior comprehensive technique than VCTS for NTC 8T-SPUFs.

\subsection{Overhead Analysis}

The techniques in CUBIT can be realized in SPUF array at the granularity of array logic and column. Like most of other circuit level techniques [3], area and power overheads of CUBIT relative to the entire SPUF array is insignificant because the area and power consumed by the SPUF cells hugely dominates the resources consumed by SPUF's array logic [15][3].

For CUSIT, we observe that CUSIT's relative sizing over VCTS's holistic sizing achieves linear savings in transistor's active area and power consumtion with size upscaling factors (Table 3). Although overheads in CUBIT inrease linearly with upscaling factors (Table 3), we can amortize them with respect to conventional 6T-SPUFs by adopting future technology nodes in design. This is because the size of 8T-SPUFs can be smaller than 6T-SPUFs along the future technology nodes, as 6T-SPUF's further shrinkability with future technology nodes is already limited by their cell stability issues $[10,14]$.

\section{RELATED WORKS}

The improvement of SPUFs has been explored in previous works with primary focus on STC operated 6T-SRAM cells. Garg et al. addresses uniformity and reliability improvement by utilizing aging effects, like application of NBTI stress [7]. This technique cannot control the gate leakage current which is primarily responsible for degraded uniformity, in an 8T-SPUF. 


\begin{tabular}{|c|c|c|c|c|c|c|c|c|c|c|}
\hline \multirow[t]{2}{*}{ Priority } & \multirow[t]{2}{*}{ RLT } & \multirow[t]{2}{*}{ ULT } & \multirow[t]{2}{*}{ Bias Set } & \multicolumn{3}{|c|}{ PUC(\%) } & \multicolumn{3}{|c|}{ Uniformity(\%) } & \multirow{2}{*}{$\begin{array}{l}\text { Enhancement } \\
(\mathrm{R}, \mathrm{U})(\mathrm{in} \%)\end{array}$} \\
\hline & & & & $-40^{\circ} \mathrm{C}$ & $25^{\circ} \mathrm{C}$ & $110^{\circ} \mathrm{C}$ & $-40^{\circ} \mathrm{C}$ & $25^{\circ} \mathrm{C}$ & $110^{\circ} \mathrm{C}$ & \\
\hline $\begin{array}{c}\text { Reliability } \\
\text { Favoring }\end{array}$ & $5 \%$ & $10 \%$ & $\begin{array}{c}\text { \{RE-RBL, RE-RWL, } \\
\text { UE-SGT0.17\} }\end{array}$ & 17.4 & 16.7 & 17.5 & 49.09 & 47.16 & 43.77 & $(55.8 \%, 81.35 \%)$ \\
\hline $\begin{array}{l}\text { Uniformity } \\
\text { Favoring }\end{array}$ & $10 \%$ & $5 \%$ & \{RE-RBL, UE-SGT0.33\} & 18.8 & 18.9 & 18.4 & 56.50 & 51.21 & 44.27 & $(52.27 \%, 82.86 \%)$ \\
\hline $\begin{array}{l}\text { Single Voltage } \\
\text { Source }\end{array}$ & $10 \%$ & $10 \%$ & \{RE-RWL, RUE-SGT $\}$ & 17.8 & 16.2 & 17.9 & 42.29 & 42.84 & 44.17 & $(54.79 \%, 76.8 \%)$ \\
\hline
\end{tabular}

Table 2: Different $(R, U)$ enhancements over base line NTC 8TSPUF, by application of different priority constraints in the CUBIT algorithm.

\begin{tabular}{|c|c|c|c|c|c|c|c|c|c|}
\hline \multirow{2}{*}{$\begin{array}{c}\text { Comparative } \\
\text { Schemes }\end{array}$} & \multirow{2}{*}{$\begin{array}{c}\text { Size } \\
\text { Upscaling }\end{array}$} & \multirow[t]{2}{*}{ (Area,Power) } & \multicolumn{3}{|c|}{ PUC(\%) } & \multicolumn{3}{|c|}{ Uniformity(\%) } & \multirow{2}{*}{$\begin{array}{l}\text { Enhancement } \\
(R, U) \text { (in \%) }\end{array}$} \\
\hline & & & $-40^{\circ} \mathrm{C}$ & $25^{\circ} \mathrm{C}$ & $110^{\circ} \mathrm{C}$ & $-40^{\circ} \mathrm{C}$ & $25^{\circ} \mathrm{C}$ & $110^{\circ} \mathrm{C}$ & \\
\hline \multirow{5}{*}{ CUSIT } & Baseline & $(1 \mathrm{x}, 1 \mathrm{x})$ & 14.8 & 22.6 & 39.6 & 83.27 & 80.10 & 73.32 & $(-,-)$ \\
\hline & Relative X2 & $(2 x, 2.15 x)$ & 13.3 & 19.0 & 30.0 & 71.02 & 67.67 & 63.50 & $(24.24,36.79)$ \\
\hline & Relative X3 & $(3 x, 3.26 x)$ & 13.0 & 17.7 & 24.4 & 65.11 & 63.04 & 60.24 & $(38.38,54.56)$ \\
\hline & Relative X4 & $(4 x, 4.38 x)$ & 12.0 & 15.6 & 21.1 & 61.70 & 60.52 & 57.89 & $(46.71,64.80)$ \\
\hline & Relative X5 & $(5 x, 5.51 x)$ & 10.7 & 14.4 & 19.5 & 59.09 & 58.56 & 56.09 & $(50.75,72.67)$ \\
\hline \multirow{5}{*}{ VCTS } & Baseline & $(1 x, 1 x)$ & 14.8 & 22.6 & 39.6 & 83.27 & 80.10 & 73.32 & $(-,-)$ \\
\hline & Holistic X2 & $(1.75 x, 2.12 x)$ & 9.9 & 15.0 & 27.1 & 82.76 & 79.31 & 72.00 & $(31.57,1.51)$ \\
\hline & Holistic X3 & $(2.5 x, 3.23)$ & 8.4 & 12.7 & 21.8 & 82.88 & 79.09 & 71.50 & $(44.95,1.16)$ \\
\hline & Holistic X4 & $(3.25 x, 4.31 x)$ & 6.7 & 11.2 & 17.3 & 82.76 & 79.29 & 71.14 & $(56.31,1.50)$ \\
\hline & Holistic X5 & $(4 x, 5.45 x)$ & 6.5 & 10.4 & 16.3 & 82.53 & 78.67 & 70.79 & $(58.83,2.21)$ \\
\hline
\end{tabular}

Table 3: Comparative analysis of Enhancement of Reliability and Uniformity

Chellapa et al. proposes an alternative SRAM cell powerup strategy, by raising the wordline and decoder voltage higher than array voltage [5]. The voltage raise defeats the key purpose of NTC operation. Chang et al. implements sizing strategies to enhance SPUF reliability, but this methodology is flawed at NTC regime [3]. A sub-threshold PUF based on the 10T-SRAM cell, introduced by Kaseem et al., is a suitable low-power solution for secure devices [11]. This parameters of reliability and uniformity have not been addressed at subtheshold operation for the 10T-SRAM PUF.

To the best of our knowledge, this is the first work which has explored reliability and uniformity characteristics for SPUFs operated at NTC and adopt efficient design strategies to overcome their adverse effects.

\section{CONCLUSION}

We analyze the various threats to reliability and uniformity characteristics of NTC-operated SPUF. Leveraging the impact of device asymmetry on these characteristics, we improvise current suppression techniques. The principles governing CUBIT and CUSIT schemes are based on biasing and sizing respectively. CUBIT and CUSIT adaptively mitigate the accentuated effects of PV on reliability and uniformity, by giving a comprehensive improvement of more than $82 \%(55 \%)$ in our reliability and uniformity metrics with negligible overheads. Thus, our work embraces on making the SPUF not only more energy-efficient but also, highly robust.

\section{REFERENCES}

[1] ASU. 2008. Predictive Technology Models (PTM) ASU. http://ptm.asu.edu/ modelcard/HP/32nm_HP.pm.

[2] Shilpi Birla, Neeraj Kumar Shukla, Kapil Rathi, Rakesh Kumar Singh, and Manisha Pattanaik. 2011. Analysis of 8T SRAM Cell at Various Process Corners at $65 \mathrm{~nm}$ Process Technology. Circuits and Systems (2011), 326-329.

[3] Chip-Hong Chang, Chao Qun Liu, Le Zhang, and Zhi Hui Kong. 2016. Sizing of SRAM Cell with Voltage Biasing Techniques for Reliability Enhancement of Memory and PUF Functions. Journal of Low Power Electronics and Applications 6, 3 (2016).

[4] L. Chang, Y. Nakamura, R. K. Montoye, J. Sawada, A. K. Martin, K. Kinoshita, F. H. Gebara, K. B. Agarwal, D. J. Acharyya, W. Haensch, K. Hosokawa, and D. Jamsek. 2007. A 5.3GHz 8T-SRAM with Operation Down to $0.41 \mathrm{~V}$ in 65nm CMOS. In 2007 IEEE Symposium on VLSI Circuits.

[5] S. Chellappa, A. Dey, and L. T. Clark. 2011. Improved circuits for microchip identification using SRAM mismatch. In 2011 IEEE Custom Integrated Circuits Conference (CICC). 1-4.

[6] Ronald G. Dreslinski, Michael Wieckowski, David Blaauw, Dennis Sylvester, and Trevor N. Mudge. 2010. Near-Threshold Computing: Reclaiming Moore's Law Through Energy Efficient Integrated Circuits. Proc. of the IEEE 98, 2 (2010), 253-266.

[7] A. Garg and T. T. Kim. 2014. Design of SRAM PUF with improved uniformity and reliability utilizing device aging effect. In 2014 IEEE International Symposium on Circuits and Systems (ISCAS). 1941-1944.

[8] C. Herder, M. D. Yu, F. Koushanfar, and S. Devadas. 2014. Physical Unclonable Functions and Applications: A Tutorial. Proc. IEEE 102 (2014).

[9] Daniel E. Holcomb, Wayne P. Burleson, and Kevin Fu. 2009. Power-Up SRAM State as an Identifying Fingerprint and Source of True Random Numbers. IEEE Trans. Computers (2009), 1198-1210.

[10] Koichiro Ishibashi. 2011. Low power and reliable SRAM memory cell and array design. Springer, Berlin New York.

[11] M. Kassem, M. Mansour, A. Chehab, and A. Kayssi. 2010. A sub-threshold SRAM based PUF. In 2010 International Conference on Energy Aware Computing. 1-4.

[12] Wiedong Liu and Chenming Hu. 2011. BSIM4 and MOSFET Modeling for IC Simulation. (2011)

[13] Abhranil Maiti, Vikash Gunreddy, and Patrick Schaumont. 2011. A Systematic Method to Evaluate and Compare the Performance of Physical Unclonable Functions. IACR Cryptology ePrint Archive 2011 (2011).

[14] Yasuhiro Morita, Hidehiro Fujiwara, Hiroki Noguchi, Yusuke Iguchi, Koji Nii, Hiroshi Kawaguchi, and Masahiko Yoshimoto. 2007. Area Optimization in 6T and 8T SRAM Cells Considering $V_{\text {th }}$ Variation in Future Processes. IEICE Transactions (2007), 1949-1956.

[15] S. Mukhopadhyay, H. Mahmoodi, and K. Roy. 2005. Modeling of failure probability and statistical design of SRAM array for yield enhancement in nanoscaled CMOS. 24, 12 (dec. 2005), 1859 - 1880. https://doi.org/10. 1109/TCAD.2005.852295

[16] P. Simons, E. van der Sluis, and V. van der Leest. 2012. Buskeeper PUFs, a promising alternative to D Flip-Flop PUFs. In 2012 IEEE International Symposium on Hardware-Oriented Security and Trust.

[17] G. Edward Suh and Srinivas Devadas. 2007. Physical unclonable functions for device authentication and secret key generation. In Proc. of DAC (DAC '07). 9-14.

[18] Synopsis. 2013. HSPICE ${ }^{\circledR}$ User Guide: Advanced Analog Simulation and Analysis. 